Discussion Paper No. 746

\title{
CHEAP TALK WITH AN INFORMED RECEIVER
}

\author{
Junichiro Ishida \\ and \\ Takashi Shimizu
}

June 2009

The Institute of Social and Economic Research Osaka University

6-1 Mihogaoka, Ibaraki, Osaka 567-0047, Japan 


\title{
Cheap Talk with an Informed Receiver*
}

\author{
Junichiro Ishida ${ }^{\dagger}$ and Takashi Shimizu ${ }^{\ddagger}$
}

June 23, 2009

\begin{abstract}
This paper examines the effectiveness of cheap talk when the receiver is imperfectly informed. We show that the receiver's prior knowledge becomes an impediment to efficient communication in a model with the discrete state space: in general, the more the receiver is informed, the less information she can extract from the sender. In fact, when the receiver is as informed as the sender, no information can be conveyed via cheap talk for an arbitrarily small preference bias. This draws sharp contrast to the conventional setup where there is always a fully separating equilibrium as long as the preference bias is sufficiently small. We relate this result to issues that are critical for organizational design, such as the allocation of decision-making authority and the span of control.
\end{abstract}

Keywords: Cheap talk, Informed receiver, Truth telling, Rational ignorance.

JEL Classification Number: D23; D82.

*This research is financially supported by Grant-in-Aid for Scientific Research from JSPS and MEXT and the Global COE (GCOE) program of Osaka University. Of course, any remaining errors are our own.

${ }^{\dagger}$ Institute of Social and Economic Research, Osaka University, 6-1 Mihogaoka, Ibaraki-shi, Osaka 567-0047 JAPAN (E-mail: jishida@iser.osaka-u.ac.jp)

${ }^{\ddagger}$ Faculty of Economics, Kansai University, 3-3-35 Yamate-cho, Suita-shi, Osaka 564-8680 JAPAN (E-mail: tshimizu@ipcku.kansai-u.ac.jp) 


\section{Introduction}

Since the seminal work of Crawford and Sobel [6], a substantial amount of attention has been paid to strategic aspects of communication between players with conflicted interests. A canonical model of this literature, which is now widely known as "cheap talk," typically has an informed sender who communicates with an uninformed receiver by sending a costless message. ${ }^{1}$ In this setting, the amount of information which can be conveyed to the receiver depends on the degree of preference congruence between the sender and the receiver: in general, more information is conveyed when the two players' preferences are aligned more closely. This original insight has been fruitfully applied to many distinct issues, not just in economics but also in other disciplines such as political science, and has now become an indispensable tool to analyze various settings which involve communication among concerned parties. $^{2}$

Despite several notable developments that have been made over the years, however, most existing works focus on the case where the sender who knows the state of nature with precision sends a message to the receiver who knows nothing about it. An unintended consequence of the conventional setup is that it naturally limits our attention to the case where the players are maximally differentiated in terms of the quality of prior information. This leaves a lot of ground yet to be covered, especially because it indeed seems very rare, if ever, to find an occasion where one possesses perfect knowledge while the other is absolutely clueless. It remains to be seen how the nature of communication alters as the information gap between the sender and the receiver shrinks. In this paper, we shed light on this aspect of cheap talk in an environment where both the sender and the receiver are (imperfectly) informed about the state of nature.

As it turns out, the fact that the receiver has some partial prior knowledge makes a world of differences. The main finding of this paper is that the receiver's prior knowledge obstructs

\footnotetext{
${ }^{1}$ Recently, several attempts are made to explicitly incorporate the cost of lying into the setting of cheap talk. Most notably, see Kartik et al. [13] and Kartik [12].

${ }^{2}$ Some notable applications of cheap talk include Farrell and Gibbons [9] and Matthews and Postlewaite [15] for bargaining (double-auction) problems, Matthews [16] and Austen-Smith [1] for political debates, and Dessein [7] for organizational design.
} 
efficient communication: the more the receiver is informed, the less information she can extract from the sender. We make this point in a model with two states, where the sender prefers one state while the receiver prefers the other. Before communication, each player, not just the sender, observes a signal which (imperfectly) reflects the true state. The sender then sends a costless message to the receiver upon observing the signal. In this setup, we show that communication cannot be informative for an arbitrarily small preference incongruence when the receiver's signal becomes as accurate as the sender's, indicating that no information can be conveyed via cheap talk between two equally informed parties. This is in sharp contrast to the conventional setup where there is always an informative equilibrium as long as the preference incongruence is sufficiently small.

There is a very simple and intuitive reason for this result. The potential cost of misreporting is that, by doing so, it generally results in a less efficient choice of action, which lowers the welfare of both players. This is more of a concern when the receiver has absolutely no idea and hence must rely on the sender's recommendation rather blindly: since the clueless receiver follows whatever the sender insists, he has an incentive to report truthfully as log as the preference bias is sufficiently small. The situation changes drastically, however, when the receiver has some prior information because the receiver can now overrule the sender's recommendation based on her own information. The problem, from the receiver's viewpoint, is that this overruling does not occur randomly: the receiver overrules the sender's recommendation when it is more likely to be wrong. The receiver thus effectively functions as a gatekeeper to sort out bad information, but this capability, or the lack of commitment not to use her own information, clearly diminishes the sender's incentive to report truthfully. Since the sender knows that he would be corrected whenever he is way off the mark, the salience of the private benefit is magnified, which renders communication less informative.

This result suggests that the overall efficiency can be non-monotonic with respect to the amount of information held by the receiver. When the receiver is less informed, communication is informative and the receiver can effectively combine the observed signals to reach a better decision. The overall efficiency continues to rise as the receiver's signal becomes more accurate, as long as communication is informative. At some point, however, communication 
becomes uninformative and the receiver must rely on her own information, at which point the overall efficiency takes a sharp spike. Past this point, the efficiency depends solely on the receiver's information and hence gradually improves as it becomes more accurate. This insight yields several critical implications for the allocation of decision-making authority as well as the optimal form of organization. We will discus them in some depth in section 4 .

Since we allow the receiver to be informed, the present setup has some inherent connection to models with multiple senders in that there exist multiple informed parties. The most notable along this line is perhaps Krishna and Morgan [14], who consider a situation where there are two senders who send messages sequentially and a receiver makes a decision. ${ }^{3}$ Our model can almost be regarded as the case where the receiver's preferences align perfectly with the second sender's, so that information extraction from the second sender is not an issue. ${ }^{4}$ The difference is that they only consider the case where both of the senders are perfectly informed, as do most of the existing works. In their setup, the case where the receiver's preferences coincide with the second sender's is roughly equivalent to the case where the receiver is perfectly informed. Krishna and Morgan [14] do not extend their analysis to that situation for the obvious reason: the case with a perfectly informed receiver is simply meaningless as there is no point in asking anyone's opinion. In contrast, if we translate our model into a setup with two senders, it corresponds to the case where both of the senders are only imperfectly informed, which makes communication with the first sender potentially valuable.

As mentioned, a critical point of departure is that the players are only imperfectly informed. While most of existing works deal with perfect information, there are some works which analyze the case with imperfectly informed players. Austen-Smith [2] considers a case where an uninformed decision maker solicit advice from two biased experts who are imperfectly informed and compares two different forums: multiple joint referral and multiple

\footnotetext{
${ }^{3}$ See also Gilligan and Krehbiel [11] and Battaglini [3].

${ }^{4}$ We say "almost" because the fact that the receiver and the second sender are the same individual, which is the case in our setup, yields a slightly different implication. In a case where the receiver's preferences align with the second sender's, it is always possible to construct an equilibrium in which the receiver totally ignores the second sender's message. When the receiver and the second sender are the same individual, however, this option is in general not available.
} 
sequential referral. Battaglini [4] further extends this analysis by considering both an arbitrary number of imperfectly informed experts and a multidimensional state space. ${ }^{5}$ Gerardi et al. [10] also consider a model with arbitrary many imperfectly informed experts where preference biases are their private information, and investigate what type of decision rule can effectively extract information from the experts. In all of those works, however, the receiver is still totally uninformed whereas in ours, we allow the receiver (the decision maker) to possess some partial knowledge about the state of nature. ${ }^{6}$

In this respect, the paper that is close to ours is Olszewski [17] who analyzes the case where the receiver is also endowed with some private information. ${ }^{7}$ The focus of his analysis differs sharply from ours, however, as it seeks for conditions under which truth-telling equilibria emerge as a unique equilibrium. To this end, he introduces a behavioral sender type who always reveals honestly and reputation concerns for being honest into a cheap-talk model. The main result is then obtained in the case where the sender is motivated purely by the reputation concerns: in his analysis, the receiver's private information facilitates communication because it enables her to discriminate truth from lie. In contrast, we consider a much simpler setting with no behavioral sender types (and hence no reputation concerns) and proceed in the opposite direction to show that the receiver's (imperfect) prior knowledge obstructs efficient communication.

The paper proceeds as follows. Section 2 provides a motivating example with two signals, hopefully to clarify what we try to get at in this paper. Section 3 extends the analysis to incorporate an arbitrary finite number of signals and shows that the basic insight obtained in section 2 holds in a more general environment. Section 4 discussed several implication of

\footnotetext{
${ }^{5}$ The fact that experts are only imperfectly informed is crucial in a setting with more than two experts, because a unilateral defection can surely be detected if experts are all perfectly informed.

${ }^{6}$ Doraszelski et al. [8] consider a different setting where there are two imperfectly informed players who must decide whether to form a partnership with uncertain rewards jointly by voting. Before voting, the players can talk to each other to exchange information about the state of nature. In this setting, communication is beneficial as it provides a double-check. The focus of attention is clearly different from ours, as their model focuses on the interaction between communication and voting.

${ }^{7}$ An alternative approach is provided by Ottaviani and Sørensen [18][19] where informed exerts are concerned with their reputation for being well informed. Prendergast [20] provides a model of "yes men" where an agent reports the best estimate of the principal's observation, rather than his own observation, when the reward scheme depends on the difference between the agent's report and the principal's observation. In a different vein, Blume et al. [5] introduce communication errors and show that adding noise can improve welfare.
} 
the model, especially on the optimal design of organization. Finally section 5 makes some concluding remarks.

\section{An Illustrative Example}

We start with a simple example with two signals to illustrate how the receiver's imperfect knowledge becomes an impediment to effective communication. There are two players, Player 1 (male) and Player 2 (female), to whom we interchangeably refer as the sender and the receiver, respectively, throughout the analysis. The game proceeds as follows:

1. Nature randomly chooses the state $t=1$ or 2 with equal probability, i.e.,

$$
P(t=1)=P(t=2)=0.5 \text {. }
$$

2. Player $n(n=1,2)$ receives a private signal $s_{n}=1$ or 2 . We assume that $s_{1}$ and $s_{2}$ are drawn independently and

$$
P\left(s_{n}=k \mid t=k\right)=r_{n} \in[0.5,1] \quad n, k=1,2 .
$$

3. Upon observing $s_{1}$, Player 1 costlessly sends a message $m=1$ or 2 to Player 2 .

4. Upon observing $s_{2}$ and $m$, Player 2 chooses an action $a=1$ or 2 .

5. Player $n$ receives a payoff $u_{n}(t, a)$ such that

$$
u_{n}(t, a)=\mathbb{I}(a=t)+b_{n} \mathbb{I}(a=n)
$$

where $\mathbb{I}$ is the indicator function, and $b_{n}$ is Player $n$ 's private benefit of implementing his or her preferred action.

In this specification, $r_{n}$ represents the accuracy of Player $n$ 's prior information, which is a measure of how informed the player is, and $r_{1}-r_{2}$ represents what we call the information gap. As usual, each player has his or her own preferred action, so that their preferences are not perfectly aligned. The degree of preference incongruence is measured by $b_{n}$, which we refer to as the preference bias for the ease of exposition. Let $\beta_{n}:=\left(1+b_{n}\right) / 2$, where we assume $b_{n} \in(0,1)$ or, equivalently, $\beta_{n} \in(0.5,1)$ for $n=1,2$. 
Throughout this paper we restrict our attention to pure-strategy equilibria. Under this premise, the sender's strategy can be written as $M\left(s_{1}\right)$ and the receiver's strategy as $A\left(m, s_{2}\right)$. We then search for an equilibrium in which "communication matters," i.e., the sender reveals his observation truthfully and the receiver's choice of action depends on his message at least with some positive probability.

We start with the problem faced by the receiver who observes her own signal $s_{2}$ and also receives a message $m$ from the sender. Define $P_{i j}:=P\left(t=1 \mid s_{1}=i, s_{2}=j\right)$. Given that the sender reports truthfully, the posterior belief is computed as

$$
\left\{\begin{array}{l}
P_{11}=\frac{r_{1} r_{2}}{r_{1} r_{2}+\left(1-r_{1}\right)\left(1-r_{2}\right)} \\
P_{12}=\frac{r_{1}\left(1-r_{2}\right)}{r_{1}\left(1-r_{2}\right)+\left(1-r_{1}\right) r_{2}}, \\
P_{21}=\frac{\left(1-r_{1}\right) r_{2}}{\left(1-r_{1}\right) r_{2}+r_{1}\left(1-r_{2}\right)} \\
P_{22}=\frac{\left(1-r_{1}\right)\left(1-r_{2}\right)}{\left(1-r_{1}\right)\left(1-r_{2}\right)+r_{1} r_{2}} .
\end{array}\right.
$$

Once the posterior belief is obtained, the receiver's best response is characterized by the following simple rule:

$$
A(i, j)= \begin{cases}1 & \text { only if } P_{i j} \geq \beta_{2} \\ 2 & \text { only if } P_{i j} \leq \beta_{2} .\end{cases}
$$

Taking the receiver's strategy $A$ as given, the sender decides what message to convey to the receiver. A necessary, and naturally more stringent, condition for truth telling is that the sender must have an incentive to disclose his observation truthfully when he observes what he does not like, i.e., $s_{1}=2$. This condition can be written as

$$
\mathbb{E}\left[u_{1}\left(t, A\left(2, s_{2}\right)\right) \mid s_{1}=2\right] \geq \mathbb{E}\left[u_{1}\left(t, A\left(1, s_{2}\right)\right) \mid s_{1}=2\right] .
$$

There exists a fully separating (truth-telling) equilibrium when this condition is satisfied.

One might conjecture that (3) is satisfied as long as the preference biases $b_{n}$ are sufficiently small. As it turns out, though, this conjecture does not necessarily hold true as the receiver becomes as informed as the sender. To see this, note first that $\lim _{r_{2} \rightarrow r_{1}} P_{12}=$ $\lim _{r_{2} \rightarrow r_{1}} P_{21}=0.5$, which implies that $A(2,2)=A(1,2)=A(2,1)=2$ for any given positive bias $b_{2}$. For communication to have any value, therefore, one must have $A(1,1)=1$, which is the case if $P_{11} \geq \beta_{2}$. Given this, (3) can be written as

$$
r_{1} \geq r_{1}\left(r_{2}+\left(1-r_{2}\right) b_{1}\right)+\left(1-r_{1}\right) r_{2}\left(1+b_{1}\right)=r_{2}+\left(r_{1}\left(1-r_{2}\right)+\left(1-r_{1}\right) r_{2}\right) b_{1}
$$


The left-hand side is the expected payoff of recommending $m=2$, while the right-hand side is that of $m=1$. As $r_{2} \rightarrow r_{1}$, this condition collapses to

$$
0 \geq 2 r_{1}\left(1-r_{1}\right) b_{1}
$$

which cannot be satisfied by any positive $b_{1}$, no matter how small it is. In fact, in this simple example, one can even make a stronger statement: with positive preference biases, communication cannot be informative for any $r_{2} \geq r_{1} .{ }^{8}$

To understand this result, one must look at, as always, the cost and benefit of misrepresenting information. First, the benefit is relatively clear, as the sender may sway the receiver towards his preferred action by making a biased recommendation. This benefit is represented by $\left(r_{1}\left(1-r_{2}\right)+\left(1-r_{1}\right) r_{2}\right) b_{1}$, which is strictly positive for any $b_{1}>0$ and $r_{n}$. This information manipulation comes at a cost, however, because it necessarily entails an inefficient use of information. Given the receiver's strategy, a potential loss arises when the receiver observes $s_{2}=1$, in which case the sender's message becomes pivotal. That is, the cost of misrepresenting information is determined by how much the sender loses by recommending his preferred action when $s_{1}=2$ and $s_{2}=1$, which is precisely captured by $P_{21}$. As the receiver's information becomes as accurate as the sender's, $P_{21}$ converges towards one half. At this point, therefore, it is a fair bet, one way or the other, as far as the probability of choosing the right action is concerned. The sender can only gain from lying by the margin of the preference bias, thereby extinguishing any hope for informative communication.

This simple example sheds light on an important aspect of communication: in a strategic situation with conflicted interests, it is increasingly difficult to exchange meaningful information when concerned parties are equally (and imperfectly) informed. In the next section, we extend this argument to a more general setting and obtain conditions under which communication ceases to work in this environment.

\footnotetext{
${ }^{8}$ Obviously, (4) never holds when $r_{2}>r_{1}$. On the other hand, as $r_{2}$ gets past $r_{1}$, we may have $P_{11}>P_{21}>$ $\beta_{2}>P_{12}>P_{22}$ at some point. In this case, the receiver's information is so accurate that she does not need to rely on the sender's information.
} 


\section{The General Model}

\subsection{Preliminary: the Setup and the Equilibrium Conditions}

We consider basically the same model as in the previous section but enrich its information structure to incorporate arbitrary finite sets of private signals. Let $S_{n}=\left\{1,2, \ldots,\left|S_{n}\right|\right\}$ denote the set of signals for Player $n$ and $\ell_{n}$ denote the likelihood ratio function where

$$
\ell_{n}(i)=\frac{P\left(s_{n}=i \mid t=1\right)}{P\left(s_{n}=i \mid t=2\right)}
$$

Without loss of generality, we order the signals by the likelihood ratio:

$$
\left\{\begin{array}{l}
\ell_{1}(1)>\ell_{1}(2)>\cdots>\ell_{1}\left(\left|S_{1}\right|\right), \\
\ell_{2}(1)>\ell_{2}(2)>\cdots>\ell_{2}\left(\left|S_{2}\right|\right) .
\end{array}\right.
$$

For the ease of exposition, we often refer to $\left(P\left(s_{n} \mid t\right)\right)_{s_{n} \in S_{n}, t=1,2}$ as Player $n$ 's signal structure. In this setup, we also allow for the possibility that $P(t=1) \neq P(t=2)$.

Since the nature of the problem virtually remains the same, the equilibrium conditions only need some slight modifications, mostly generalizations, from the two-signal example. Given the sender's strategy, define

$$
\mathcal{S}_{1}(m)=\left\{i \in S_{1} \mid M(i)=m\right\}
$$

as the set of the sender's types sending a message $m$ on the equilibrium path. With this definition, we can obtain a generalized version of (2).

Lemma 1 Given the sender's strategy $M$, the receiver's best response is

$$
A(m, j)= \begin{cases}1 & \text { if } \frac{\sum_{i \in \mathcal{S}_{1}(m)} P_{i j} P\left(s_{1}=i \mid s_{2}=j\right)}{\sum_{i \in \mathcal{S}_{1}(m)} P\left(s_{1}=i \mid s_{2}=j\right)}>\beta_{2}, \\ 2 & \text { if } \frac{\sum_{i \in \mathcal{S}_{1}(m)} P_{i j} P\left(s_{1}=i \mid s_{2}=j\right)}{\sum_{i \in \mathcal{S}_{1}(m)} P\left(s_{1}=i \mid s_{2}=j\right)}<\beta_{2} .\end{cases}
$$

Proof: See Appendix.

Similarly, we also investigate the sender's problem to obtain a generalized version of (3). To this end, define

$$
\mathcal{S}_{2}\left(i, i^{\prime}\right)=\left\{j \in S_{2} \mid A(M(i), j)=2, A\left(M\left(i^{\prime}\right), j\right)=1\right\},
$$


as the set of signals for which the receiver changes her choice of action in response to the sender's message on the equilibrium path. We then obtain the following result.

Lemma 2 If the receiver's strategy $A$ satisfies (6), then for any $i, i^{\prime} \in S_{1}$, either one of the followings holds:

(i) $\mathcal{S}_{2}\left(i, i^{\prime}\right)=\mathcal{S}_{2}\left(i^{\prime}, i\right)=\emptyset$.

(ii) $\mathcal{S}_{2}\left(i, i^{\prime}\right) \neq \emptyset$ and $\mathcal{S}_{2}\left(i^{\prime}, i\right)=\emptyset$.

(iii) $\mathcal{S}_{2}\left(i, i^{\prime}\right)=\emptyset$ and $\mathcal{S}_{2}\left(i^{\prime}, i\right) \neq \emptyset$.

Moreover,

$$
\mathbb{E}\left[u_{1}(t, A(M(i), j)) \mid s_{1}=i\right] \geq \mathbb{E}\left[u_{1}\left(t, A\left(M\left(i^{\prime}\right), j\right)\right) \mid s_{1}=i\right]
$$

if and only if

$$
\left\{\begin{array}{l}
\text { (i) holds, } \\
\text { (ii) and } \beta_{1} \leq \frac{\sum_{j \in \mathcal{S}_{2}\left(i, i^{\prime}\right)}\left(1-P_{i j}\right) P\left(s_{2}=j \mid s_{1}=i\right)}{\sum_{j \in \mathcal{S}_{2}\left(i, i^{\prime}\right)} P\left(s_{2}=j \mid s_{1}=i\right)} \text { hold, or } \\
\text { (iii) and } \beta_{1} \geq \frac{\sum_{j \in \mathcal{S}_{2}\left(i^{\prime}, i\right)}\left(1-P P_{i j}\right) P\left(s_{2}=j \mid s_{1}=i\right)}{\sum_{j \in \mathcal{S}_{2}\left(i^{\prime}, i\right)} P\left(s_{2}=j \mid s_{1}=i\right)} \text { hold. }
\end{array}\right.
$$

Proof: See Appendix.

These results straightforwardly lead to the conditions that must be satisfied by any equilibrium in this generalized setting.

Proposition $1(M, A)$ constitutes an equilibrium if and only if

- (6) holds for any $m \in M$ such that $\mathcal{S}_{1}(m) \neq \emptyset$ and any $j \in S_{2}$, and

- (7) holds for any $i, i^{\prime} \in S_{1}$.

\subsection{Fully Separating Equilibria}

In this setup with the discrete state space, one may conjecture that perfect revelation of information is feasible as long as the preference biases $b_{n}$ are sufficiently small. As we have seen 
in the example above, however, this conjecture is not generally true: no separating equilibria exist in the two-signal example when the signal structures satisfy a certain condition, namely $r_{2}$ being sufficiently close to $r_{1}$. We now extend this argument to this generalized setting and obtain more general conditions under which (fully separating) truth-telling equilibria fail to exist.

As in the two-signal example, we continue to restrict our attention to equilibria in which "communication matters" or, more formally, what we call informative equilibria defined as follows.

Definition 1 An equilibrium is truth telling if $M(i)=i$ for any $i \in S_{1}$.

Definition 2 The sender's message is influential on $j$ if there exist $m$ and $m^{\prime}$ such that $A(m, j)=2$ and $A\left(m^{\prime}, j\right)=1$, influential if it is influential on some $j$, and uninfluential if it is not influential on any $j$.

Definition 3 An equilibrium is fully informative if it is truth telling and the sender's message is influential.

The distinction between truth-telling and (fully) informative equilibria is not substantial. We nonetheless need this distinction because we can in principle have a truth-telling equilibrium in which the sender's message is uninfluential, i.e., the receiver acts on her own information alone without ever relying on the sender's message. When the message is uninfluential, any strategy $M$ is weakly optimal and we can trivially construct a truth-telling equilibrium. The definition of informative equilibria rules out this apparently uninteresting situation.

Given these definitions, we can make the following statement which is our main result.

Condition 1 Each $j \in S_{2}$ satisfies, at least, one of the followings:

(i) there exists $i(j) \in S_{1}$ such that $1-\beta_{1}<P_{i(j) . j}<\beta_{2}$,

(ii) $P_{i j}>\beta_{2} \quad \forall i$, or 
(iii) $P_{i j}<\beta_{2} \quad \forall i$.

Theorem 1 Under Condition 1, there exist no fully informative equilibria.

Proof: See Appendix.

When a signal $j$ satisfies either (ii) or (iii) of Condition 1 , the receiver's own signal is so accurate that there is no need to rely on the sender's information. When every signal $j \in S_{2}$ satisfies either (ii) or (iii), the receiver's choice of action is totally independent of the seder's message and any $M$, including the truth-telling one, is hence weakly optimal for the sender. This is apparently a trivial and uninteresting case since communication actually plays no role.

Things become more intriguing, therefore, when there exists some $j \in S_{2}$ for which neither (ii) nor (iii) holds: this is the case where the sender's message can be influential and communication plays some role. Even in this case, however, perfect revelation of information cannot be attained if that signal satisfies (i). This is a situation where the sender's message roughly cancels out the receiver's signal, so that the resulting posterior belief falls into the range where the conflict of interest between the sender and the receiver is at its pinnacle: when the posterior belief falls into this intermediate range, the players can never agree on which action to take even with complete information. Theorem 1 shows that with this extreme preference incongruence, it is impossible to extract truthful information from the sender.

To understand Theorem 1 more intuitively, it is instructive to consider the two-signal example presented in the previous section. As we have already seen, no informative equilibria exist in this example when $r_{1}=r_{2}$. We now show that the signal structures in this example satisfy Condition 1, which can best be seen graphically by Figure $1 .{ }^{9}$ In this example, $S_{2}=\{1,2\}$ and Condition 1 must be satisfied for each $s_{2} \in S_{2}$ : first, $s_{2}=1$ satisfies (i) because there is a signal $s_{1}=2$ with the corresponding posterior belief $P_{21}$ that falls into the intermediate range; second, $s_{2}=2$ satisfies (iii) for $P_{i 2}<\beta_{2}$ for any $i$. This means that Condition 1 is satisfied in this example and, in light of Theorem 1, no informative equilibria

\footnotetext{
${ }^{9}$ In the figure, we set $r_{1}=r_{2}=0.75$ and $b_{1}=b_{2}=0.2$.
} 
exist. A critical part of this example is that the opposite signals exactly cancel out each other, so that $P_{12}=P_{21}=0.5$. When that happens, as can be seen from the figure, Condition 1 is satisfied for any strictly positive preference bias $b_{n}>0$

Two features of the signal structures are at work to make this particular example work. First, in the two-signal example, the signal structures are by design "symmetric." Second, holding this symmetry, Condition 1 holds for any given $b_{n}>0$ when $r_{2}$ is sufficiently close to $r_{1}$. One can then deduce from these that Condition 1 holds when the signal structures are sufficiently "symmetric" and "similar." Below, we formalize and validate this conjecture.

Definition 4 Player 1's signal structure is symmetric if for any $i \leq \frac{\left|S_{1}\right|+1}{2}$,

$$
\ell_{1}(i) \cdot \ell_{1}\left(\left|S_{1}\right|+1-i\right)=1
$$

Definition 5 Player 2's signal structure is $\delta$-close to Player 1's if $\left|S_{1}\right|=\left|S_{2}\right|$ and

$$
\min _{t, i}\left\|P\left(s_{2}=i \mid t\right)-P\left(s_{1}=i \mid t\right)\right\|<\delta
$$

Proposition 2 Consider the environment in which $P(t=1)=P(t=2)$ and Player 1's signal structure is symmetric. Then there exists $\bar{\delta}>0$ such that for any $\delta \in(0, \bar{\delta})$, if Player 2's information is $\delta$-close to Player 1's, then Condition 1 holds.

Proof: Define $i(j)=\left|S_{1}\right|+1-j$. Then $P_{i(j) . j} \rightarrow 0.5$ as $\delta \rightarrow 0$. Therefore we can find a suitable $\bar{\delta}$.

Clearly, (i) of Condition 1 is crucial for the existence of informative equilibria. By approaching from the opposite side, we can obtain a condition under which informative equilibria always exist.

Condition 2 Both of the following conditions hold:

(i) for each $j \in S_{2}$, there exits no $i$ such that $1-\beta_{1} \leq P_{i j} \leq \beta_{2}$, and

(ii) there exist $j, i$, and $i^{\prime}$ such that $P_{i j}<1-\beta_{1}$ and $P_{i^{\prime} j}>\beta_{2}$. 
Theorem 2 Under Condition 2, there always exist fully informative equilibria.

Proof: See Appendix.

To see this result, we again go back to the two-signal example and consider the canonical case of a totally uninformed receiver. The fact that the receiver is totally uninformed is equivalent to $r_{2}=0.5$ in the two-signal example, i.e., the signals are not at all informative. As one can easily confirm, in this setup, informative equilibria always exist as long as the preference biases are sufficiently small. If the sender's signal is informative even slightly, i.e., $r_{1}>0.5$, then $P_{s_{1} s_{2}} \neq 0.5$ for any $\left(s_{1}, s_{2}\right)$. Then, as $b_{n} \rightarrow 0$ and hence $\beta_{n} \rightarrow 0.5$, Condition 2 is bound to hold at some point. This leads to the outcome we are all accustomed to: with the discrete state space, perfect revelation of information via cheap talk is feasible as long as the preferences are sufficiently congruent. This is illustrated in Figure 2: in the figure, we use the same parameters as in Figure 1, except that we set $r_{2}=0.5$. In this case, the corresponding posterior belief $P_{i j}$ lies outside of either $\beta_{2}$ or $1-\beta_{1}$ for each $j \in S_{2}$, so that Condition 2 is satisfied.

\subsection{Hybrid Equilibria}

While perfect revelation of information is not feasible in a non-trivial way under Condition 1 , this does not necessarily mean that communication is of no use because one can still convey some, though coarser, information by combining several signals. In what follows, we extend our analysis to this class of (partially pooling) hybrid equilibria and examine its properties.

It may appear that when the number of signals is large, the analysis of hybrid equilibria becomes hopelessly tedious with so many different combinations of signals. The following result establishes, however, that we only need to look at a particular class of equilibria, which simplifies the analysis to some extent.

Definition 6 An equilibrium $(M, A)$ is partitioned if there exists a partition $\left\{I_{1}, \ldots, I_{K}\right\}$ of $S_{1}$ with the following properties:

- there exist distinctive $m_{1}, \ldots, m_{K}$ such that $i \in I_{k} \Rightarrow M(i)=m_{k}$, 
Table 1: Player $n$ 's signal structure

\begin{tabular}{|c|c|c|c|}
\hline & $s_{n}=1$ & $s_{n}=2$ & $s_{n}=3$ \\
\hline$t=1$ & $q r$ & $1-q$ & $q(1-r)$ \\
\hline$t=2$ & $q(1-r)$ & $1-q$ & $q r$ \\
\hline
\end{tabular}

- $k<k^{\prime} \Rightarrow i<i^{\prime} \quad \forall i \in I_{k}, \forall i^{\prime} \in I_{k^{\prime}}$, and

- $k<k^{\prime} \Rightarrow$ there exists some $j$ such that $A\left(m_{k}, j\right)=1, A\left(m_{k^{\prime}}, j\right)=2$.

Proposition 3 If there exists an equilibrium $(M, A)$, then there also exists a corresponding partitioned equilibrium $\left(M^{*}, A^{*}\right)$ which realizes the same allocation in the sense that $A(M(i), j)=A^{*}\left(M^{*}(i), j\right)$ for any $i \in S_{1}$ and $j \in S_{2}$.

Proof: See Appendix.

This result allows us to focus on the class of partitioned equilibria, amongst a plethora of all plausible hybrid equilibria, without loss of generality. Given this, we first show that in order to obtain an analogous result to Theorem 1 for hybrid equilibria, Condition 1 is not sufficient. This is illustrated by the following example which satisfies the condition.

Example: Consider the following environment:

- $P(t=1)=P(t=2)=1 / 2$.

- $\left|S_{1}\right|=\left|S_{2}\right|=3$.

- Player $n$ 's signal structure is depicted in Table 1 , where $r \in[1 / 2,1), q \in(0,1)$.

In this example, since Condition 1 holds, there exist no informative equilibria. With more than two signals, however, there may still be a way to convey information by arbitrarily combining nearby signals. To see this let the partition be given by $I_{1}=\{1,2\}$ and $I_{2}=\{3\}$. This partition of the signals can credibly convey some information if and only if the following 
$(M, A)$ constitutes an equilibrium:

$$
A(m, j)= \begin{cases}1 & \text { if } m=M(1)=M(2) \text { and } j=1,2 \\ 2 & \text { otherwise }\end{cases}
$$

The equilibrium condition for such $(M, A)$ is

$$
\begin{aligned}
& \beta_{1} \leq \frac{\sum_{j=1,2}\left(1-P_{3 j}\right) P\left(s_{2}=j \mid s_{1}=3\right)}{\sum_{j=1,2} P\left(s_{2}=j \mid s_{1}=3\right)} \\
& \beta_{2} \leq \frac{\sum_{i=1,2}\left(1-P_{i 2}\right) P\left(s_{1}=i \mid s_{2}=2\right)}{\sum_{i=1,2} P\left(s_{1}=i \mid s_{2}=2\right)}
\end{aligned}
$$

These inequalities are rewritten as

$$
\begin{aligned}
& \beta_{1} \leq \frac{q r(1-r)+(1-q) r}{2 q r(1-r)+1-q} \\
& \beta_{2} \leq \frac{1-q+q r}{2-q}
\end{aligned}
$$

One can easily show that there exists $(r, q)$ satisfying the above inequalities: for example let $r=1-\epsilon^{2}$ and $1-\epsilon$, then under any sufficiently small $\epsilon>0$, the above inequalities hold.

This example shows that more stringent conditions are in general needed to rule out hybrid equilibria altogether. This is due to the fact that even if the sender's signal structure is sufficiently "symmetric" to begin with, this symmetry can easily be broken by arbitrarily bundling some nearby signals into a single message. Condition 1 needs to be modified to rule out any hybrid equilibria. The following is a sufficient condition for communication to have no value at all in the current setup.

Condition 3 There exist $\hat{i} \in S_{1}-\left\{\left|S_{1}\right|\right\}$ and $\hat{j} \in S_{2}-\left\{\left|S_{2}\right|\right\}$ such that

$$
\begin{aligned}
\beta_{2}>P_{i j}>1-\beta_{1} \text { for } i & >\hat{i} \text { and } j \leq \hat{j} \text {, or } \\
i & \leq \hat{i} \text { and } j>\hat{j} .
\end{aligned}
$$

Theorem 3 Under Condition 3, there exist no equilibria in which the sender's message is influential.

Proof: See Appendix. 


\section{Implications}

In most organizations, information is typically dispersed among different individuals, and it is hence imperative to somehow find a way to aggregate it. In particular, our results show that the distribution of information within an organization significantly affects the extent to which communication can be an effective tool to exchange information. While we have seen this in an environment where the distribution of information is given exogenously, this can easily be made endogenous. First, in our setup, the receiver is the decision maker who holds authority to make a final decision. For any given distribution of information, therefore, an organization designer may control the flow of information to some extent by appropriately choosing the decision maker. Second, the quality of information held by each party itself can also be endogenous, depending possibly on how much effort to exert to collect information. In this section, we focus on these two aspects in turn to draw more practical implications of our results.

\subsection{The Allocation of Authority: Hierarchy vs Committee}

Our model indicates that communication cannot be effective when the players are equally informed about the state of nature. This implies that the size of the information gap is a critical determinant of the allocation of final decision-making authority. An important implication to be drawn is that decision-making authority should not necessarily be given to the more informed: when the value of communication is sufficiently high, it might as well be the less informed who should hold decision-making authority.

To illustrate this point, consider a modified version of the two-signal example. A modification is made to introduce a gain from information aggregation (combining the signals), which is absent in the example. ${ }^{10}$ Suppose that the receiver, on top of the choice of action, can choose whether to make an investment to increase the value of the project. Let $h \in\{0,1\}$ denote the investment level, where the cost of the investment is given by $c>0$. The receiver's

\footnotetext{
${ }^{10}$ In general, the receiver can reach a better decision by combining the sender's observation with her own. This benefit is, however, absent in the two-signal example presented in section 2: with two signals and two actions, there exist no truth-telling equilibria in which the receiver uses both of the signals.
} 
payoff is now given by

$$
u_{2}(t, a, h)=V(h) \mathbb{I}(a=t)+b_{2} \mathbb{I}(a=n)-c h,
$$

where $V(1)=v>V(0)=1$ while the sender's payoff remains the same as above. In this setup, the investment pays off if and only if the probability of choosing the right action exceeds a certain threshold $\bar{p}$ satisfying

$$
v \bar{p}-c=\bar{p} \Leftrightarrow \bar{p}=\frac{c}{v-1} .
$$

We assume $P_{11} \geq \bar{p}>\max \left\{r_{1}, r_{2}\right\}$, so that the receiver chooses to invest if and only if her observed signal coincides with the sender's message. To simplify the analysis, we let $b=b_{1}=b_{2}$ where $b$ is assumed to be sufficiently small. In this setup, we explore the optimal allocation of decision-making authority, i.e., the one that maximizes the joint payoff $u_{1}+u_{2}$.

It is easy to see that the nature of the problem would not be affected by this modification. First, since the payoff function remains the same, it is clear that, taking the receiver's strategy as given, the sender's optimal strategy would not be affected by this modification. As for the receiver's strategy, note that when $m \neq s_{2}$, then $h=0$ regardless of the choice of action, and the problem hence remains exactly the same. The nature of the problem changes slightly when $m=s_{2}$, in which case the investment level depends on the choice of action. Even in this case, though, the receiver's optimal strategy would not be affected when $P_{11} \geq \beta_{2}$, which is exactly the case of our interest. To see this, $A(1,1)=1$ only if

$$
v P_{11}-c \geq 1-P_{11}+b_{2}
$$

which holds as long as $P_{11} \geq \beta_{2} \cdot{ }^{11}$ Under the maintained assumptions, therefore, the conclusions obtained in the two-signal example remain valid even with this modification.

Given this, we now examine how the choice of the receiver (the decision maker) affects the overall efficiency. Suppose first that the information gap is sufficiently small, so that communication cannot be informative anyway, regardless of which player becomes the receiver (the decision maker). In this case, any decision must be made based purely on the receiver's

\footnotetext{
${ }^{11}$ Note that $P_{11} \geq \beta_{2}$ implies $P_{11} \geq 1-P_{11}+b_{2}$ and $P_{11} \geq \bar{p}$ implies $v P_{11}-c \geq P_{11}$. Combining these two facts yields $v P_{11}-c \geq P_{11} \geq 1-P_{11}+b_{2}$.
} 
information. Since information cannot be aggregated in this case, it is clear that decisionmaking authority should be given to the more informed player, i.e., Player 1, who can choose the right action with higher probability. The expected joint payoff in this case is $2 r_{1}$.

Now suppose that the information gap becomes large, so that it is possible to exchange information via communication. Even in this case, if Player 1, the more informed party, is appointed to be the receiver, communication cannot be informative as we have seen in section 2. We hence end up with the same outcome as above, where the expected joint payoff is $2 r_{1}$. If Player 2 becomes the receiver, on the other hand, she can solicit information from Player 1 via communication. The expected joint payoff is $r_{1} r_{2}(1+v)+2 r_{1}\left(1-r_{2}\right)$, which is strictly larger than $2 r_{1}$ as long as $v>1$.

This simple example illuminates a case where decision-making authority should be given to the less informed party. This is more likely when the information gap is sufficiently large - a situation often observed in hierarchies where people higher up in the ladder are not necessarily more informed about the current situation. In a situation like this, it is not only acceptable for superiors to know less than subordinates but even desirable because that facilitates communication between them. This aspect of communication offers another insight which we will discuss next.

In contrast, when concerned parties are similarly informed, effective communication cannot be expected along dimensions where there is a conflict of interests. This type of situation is more likely to be observed when decisions are made by committees which often consist of relatively homogeneous and equally informed parties. If decisions are to be made by a committee, therefore, the chair of the committee must be the most informed one, which allows him/her to utilize the best available information in case the conflict of interests becomes so severe that not much can be extracted from its members. ${ }^{12}$

\footnotetext{
${ }^{12}$ Of course, that communication cannot be effective does not mean that a committee consisting of similarly informed parties is meaningless, because our analysis only considers cases where preference biases are present and salient. Communication is always informative when there is no conflict of interests, and a committee of homogenous parties can be effective in aggregating information in that case. In this environment, however, it does not matter who has decision-making authority. It is hence weakly optimal to confer authority to the more informed.
} 


\subsection{Rational Ignorance and the Span of Control}

Our results show that it is imperative to keep the receiver sufficiently ignorant about the underlying situation in order to facilitate communication. To obtain this result, we have thus far assumed that the quality of information held by each party is exogenously given and, more importantly, publicly known. In more realistic cases, though, the quality of information can be endogenous and only privately known. In that case, the receiver might be tempted to collect information on her own, but the possibility that she may resort to that option could severely limit her ability to extract information from the sender. Unless the receiver is more adept at acquiring information than the sender by a significant margin, she might as well do nothing, rather than try to obtain imperfect information. In a situation like this, therefore, the receiver must somehow find a way to credibly commit herself to remaining ignorant to extract any useful information from the sender.

To put this idea in context, we extend the modified example in section 4.1 by further adding the information-acquisition stage (while we instead fix Player 2 as the receiver). Suppose that at the beginning, the receiver has a chance to acquire information by incurring some cost $d$, while her choice is not observable to the sender. If the receiver chooses to do so, then the accuracy of her signal is given by $r_{2}=r>0.5$; if not, the signal contains no information, i.e., $r_{2}=0.5$. As above, the accuracy of the sender's signal is exogenous and we let $r_{1}=r$. Moreover, assume that $P_{11} \geq \bar{p}>r$, so that there is a gain from information aggregation.

It is straightforward to verify that if $d$ is sufficiently small, it is optimal for the receiver to acquire information, regardless of whether the sender reveals his observation truthfully or not: that is, acquiring information is a dominant strategy. Given this, the sender has no incentive to reveal his observation truthfully, making communication totally ineffective. The unique Nash equilibrium of this game is that the receiver acquires information at her own cost and the sender sends an uninformative babbling message, which is clearly an inefficient outcome. While any organization would hope to avoid this dilemma, the problem is more deep-rooted than it appears, because it is in principle much harder to prove one's ignorance. 
To overcome this problem, a hierarchical organization must be designed to assure that those higher up in the hierarchy, with decision-making authority, credibly remain ignorant. This can be achieved, for instance, if there is a way to raise the cost of information acquisition in some visible way. While there are possibly many means to achieve this end, one way is to let the decision maker oversee many subordinates in order to keep her too busy to get into every single decision to be made. This is typical of most hierarchical organizations where a single manager supervises several subordinates each of whom has expertise in his own jurisdiction. A wider span of control can be seen as a visible and credible way to keep decision makers away from obtaining imperfect information. We argue that this aspect of our model sheds some light on the pyramidal nature of hierarchies, pertaining to most organizations, and exploring this issue in this light may provide some new insight.

\section{Conclusion}

This paper explores the extent to which cheap-talk communication can credibly convey meaningful information when the receiver is partially informed. As it turns out, the receiver's prior knowledge matters and makes a non-trivial difference in the quality of information that can be extracted from the sender. As a generally rule, communication becomes less and less efficient as the receiver becomes more informed. This result yields a critical implication: in order to facilitate communication, it may be advisable for the receiver to refrain herself from acquiring her own information, even if it can be done with a relatively small cost.

The main purpose of this paper is to shed some light on an aspect of communication which, in our view, has not received enough attention thus far. This paper provides a small step towards this end, but we believe that there is much more to be done on this issue. For instance, we work with a model with the discrete space, which has its own costs and benefits. A benefit of working with this "discrete" model is that it can illuminate the impact of the receiver's prior knowledge on the quality of communication, as it can be exemplified most clearly by the two-signal example. There is also a cost, however: one drawback is that the analysis becomes increasingly intractable once we allow for the possibility of hybrid equilibria. We chose the current specification to make our point, that the receiver's prior 
knowledge matters, as clearly and emphatically as possible. To obtain a full characterization of equilibria, though, a model with the continuous state space, perhaps some variant of Crawford and Sobel [6], may serve the purpose better. It remains to be seen how far one can push the current logic in a "continuous" model. 


\section{References}

[1] David Austen-Smith. Information transmission in debate. American Journal of Political Science, 34(1):125-152, 1990.

[2] David Austen-Smith. Interested experts and policy advice: Multiple referrals under open rule. Games and Economic Behavior, 5(1):3-43, 1993.

[3] Marco Battaglini. Multiple referrals and multidimensional cheap talk. Econometrica, 70(4):1379-1401, 2002.

[4] Marco Battaglini. Policy advide with imperfectly informed experts. Advances in Theoretical Economics, 4(1):Article 1, 2004.

[5] Andreas Blume, Oliver J. Board, and Kohei Kawamura. Noisy talk. Theoretical Economics, 2:395-440, 2007.

[6] Vincent P. Crawford and Joel Sobel. Strategic information transmission. Econometrica, 50(6):1431-1451, 1982.

[7] Wouter Dessein. Authority and communication in organizations. Review of Economic Studies, 69:811-838, 2002.

[8] Ulrich Doraszelski, Dino Gerardi, and Francesco Squintani. Communication and voting with double-sided information. Contributions to Theoretical Economics, 3(1):Article 6, 2003.

[9] Joseph Farrell and Robert Gibbons. Cheap talk can matter in bargaining. Journal of Economic Theory, 48(1):221-237, 1989.

[10] Dino Gerardi, Richard McLean, and Andrew Postlewaite. Aggregation of expert opinions. Games and Economic Behavior, 65(2):339-371, 2009.

[11] Thomas Gilligan and Keith Krehbiel. Asymmetric information and legislative rules with a heterogeneous committee. American Journal of Political Science, 33(2):459-490, 1989. 
[12] Navin Kartik. Strategic communication with lying costs. forthcoming in Review of Economic Studies, 2008.

[13] Navin Kartik, Marco Ottaviani, and Francesco Squintani. Credulity, lies and costly talk. Journal of Economic Theory, 134(1):93-116, 2007.

[14] Vijay Krishna and John Morgan. A model of expertise. Quarterly Journal of Economics, 116:747-775, 2001.

[15] Steven Matthews and Andrew Postlewaite. Pre-play communication in two-person sealed-bid auctions. Journal of Economic Theory, 48(1):245-252, 1989.

[16] Steven A. Matthews. Veto threats: Rhetoric in a bargaining game. Quarterly Journal of Economics, 104:347-369, 1989.

[17] Wojciech Olszewski. Informal communication. Journal of Economic Theory, 117:180200, 2004.

[18] Marco Ottaviani and Peter Norman Sørensen. Professional advice. Journal of Economic Theory, 126(1):120-142, 2006.

[19] Marco Ottaviani and Peter Norman Sørensen. Reputational cheap talk. Rand Journal of Economics, 37(1):155-175, 2006.

[20] Canice Prendergast. A theory of "yes men". American Economic Review, 83(4):757-770, 1993. 


\section{Appendix}

Proof of Lemma 1: We have

$$
\begin{aligned}
& \mathbb{E}\left[u_{2}(t, 1) \mid s_{1} \in \mathcal{S}_{1}(m), s_{2}=j\right]-\mathbb{E}\left[u_{2}(t, 2) \mid s_{1} \in \mathcal{S}_{1}(m), s_{1}=j\right] \\
& =P\left(t=1 \mid s_{1} \in \mathcal{S}_{1}(m), s_{2}=j\right)\left(1-b_{2}\right)-P\left(t=2 \mid s_{1} \in \mathcal{S}_{1}(m), s_{2}=j\right)\left(1+b_{2}\right) \\
& =2\left[P\left(t=1 \mid s_{1} \in \mathcal{S}_{1}(m), s_{2}=j\right)-\beta_{2}\right],
\end{aligned}
$$

where

$$
\begin{aligned}
& P\left(t=1 \mid s_{1} \in \mathcal{S}_{1}(m), s_{2}=j\right) \\
& =\frac{P\left(t=1, s_{1} \in \mathcal{S}_{1}(m), s_{2}=j\right)}{P\left(s_{1} \in \mathcal{S}_{1}(m), s_{2}=j\right)} \\
& =\frac{\sum_{i \in \mathcal{S}_{1}(m)} P\left(t=1, s_{1}=i, s_{2}=j\right)}{\sum_{i \in \mathcal{S}_{1}(m)} P\left(s_{1}=i, s_{2}=j\right)} \\
& =\frac{\sum_{i \in \mathcal{S}_{1}(m)} \frac{P\left(t=1, s_{1}=i, s_{2}=j\right)}{P\left(s_{1}=i, s_{2}=j\right)} P\left(s_{1}=i, s_{2}=j\right)}{\sum_{i \in \mathcal{S}_{1}(m)} P\left(s_{1}=i, s_{2}=j\right)} \\
& =\frac{\sum_{i \in \mathcal{S}_{1}(m)} P_{i j} P\left(s_{1}=i, s_{2}=j\right)}{\sum_{i \in \mathcal{S}_{1}(m)} P\left(s_{1}=i, s_{2}=j\right)} \\
& =\frac{\sum_{i \in \mathcal{S}_{1}(m)} P_{i j} P\left(s_{1}=i \mid s_{2}=j\right)}{\sum_{i \in \mathcal{S}_{1}(m)} P\left(s_{1}=i \mid s_{2}=j\right)} .
\end{aligned}
$$

Proof of Lemma 2: To prove the lemma, we first establish the following results.

Lemma 3 For any nonempty $\tilde{S}_{1} \subseteq S_{1}$ and any $j<j^{\prime}$,

$$
\frac{\sum_{i \in \tilde{S}_{1}} P_{i j} P\left(s_{1}=i \mid s_{2}=j\right)}{\sum_{i \in \tilde{S}_{1}} P\left(s_{1}=i \mid s_{2}=j\right)}>\frac{\sum_{i \in \tilde{S}_{1}} P_{i j^{\prime}} P\left(s_{1}=i \mid s_{2}=j^{\prime}\right)}{\sum_{i \in \tilde{S}_{1}} P\left(s_{1}=i \mid s_{2}=j^{\prime}\right)} .
$$


Proof: We obtain

$$
\begin{aligned}
& \frac{\sum_{i \in \tilde{S}_{1}} P_{i j} P\left(s_{1}=i \mid s_{2}=j\right)}{\sum_{i \in \tilde{S}_{1}} P\left(s_{1}=i \mid s_{2}=j\right)}-\frac{\sum_{i \in \tilde{S}_{1}} P_{i j^{\prime}} P\left(s_{1}=i \mid s_{2}=j^{\prime}\right)}{\sum_{i \in \tilde{S}_{1}} P\left(s_{1}=i \mid s_{2}=j^{\prime}\right)} \\
& =\frac{\sum_{i \in \tilde{S}_{1}} P_{i j} P\left(s_{1}=i, s_{2}=j\right)}{\sum_{i \in \tilde{S}_{1}} P\left(s_{1}=i, s_{2}=j\right)}-\frac{\sum_{i \in \tilde{S}_{1}} P_{i j^{\prime}} P\left(s_{1}=i, s_{2}=j^{\prime}\right)}{\sum_{i \in \tilde{S}_{1}} P\left(s_{1}=i, s_{2}=j^{\prime}\right)} \\
& =\frac{1}{\left(\sum_{i \in \tilde{S}_{1}} P\left(s_{1}=i, s_{2}=j\right)\right)\left(\sum_{i \in \tilde{S}_{1}} P\left(s_{1}=i, s_{2}=j^{\prime}\right)\right)} \\
& \quad \times\left\{\sum_{i \in \tilde{S}_{1}}\left(P_{i j}-P_{i j^{\prime}}\right) P\left(s_{1}=i, s_{2}=j\right) P\left(s_{1}=i, s_{2}=j^{\prime}\right)\right. \\
& \quad+\sum_{i, i^{\prime} \in \tilde{S}_{1}, i<i^{\prime}}\left[\left(P_{i j}-P_{i^{\prime} j^{\prime}}\right) P\left(s_{1}=i, s_{2}=j\right) P\left(s_{1}=i^{\prime}, s_{2}=j^{\prime}\right)\right. \\
& \left.\left.\quad+\left(P_{i^{\prime} j}-P_{i j^{\prime}}\right) P\left(s_{1}=i^{\prime}, s_{2}=j\right) P\left(s_{1}=i, s_{2}=j^{\prime}\right)\right]\right\} .
\end{aligned}
$$

It is easily verified that $P_{i j}>P_{i j^{\prime}}$ by (5). Also, for any $i<i^{\prime}$,

$$
\begin{aligned}
& \left(P_{i j}-P_{i^{\prime} j^{\prime}}\right) P\left(s_{1}=i, s_{2}=j\right) P\left(s_{1}=i^{\prime}, s_{2}=j^{\prime}\right)+\left(P_{i^{\prime} j}-P_{i j^{\prime}}\right) P\left(s_{1}=i^{\prime}, s_{2}=j\right) P\left(s_{1}=i, s_{2}=j^{\prime}\right) \\
& =P(t=1) P(t=2) P\left(s_{2}=j \mid t=2\right) P\left(s_{2}=j^{\prime} \mid t=2\right) \\
& \quad \times\left[P\left(s_{1}=i \mid t=1\right) P\left(s_{1}=i^{\prime} \mid t=2\right)+P\left(s_{1}=i^{\prime} \mid t=1\right) P\left(s_{1}=i \mid t=2\right)\right]\left[\ell_{2}(j)-\ell_{2}\left(j^{\prime}\right)\right] .
\end{aligned}
$$

This is positive by (5) which proves the lemma.

Lemma 4 Given the receiver's strategy $A$ satisfying (6), if $A(M(i), j)=2$ and $A\left(M\left(i^{\prime}\right), j\right)=$ 1 , then there exists no $j^{\prime}$ such that $A\left(M(i), j^{\prime}\right)=1$ and $A\left(M\left(i^{\prime}\right), j^{\prime}\right)=2$.

Proof: Suppose to the contrary that there exist $\left(i, i^{\prime}, j, j^{\prime}\right)$ such that $A(M(i), j)=2$, $A\left(M\left(i^{\prime}\right), j\right)=1, A\left(M(i), j^{\prime}\right)=1$ and $A\left(M\left(i^{\prime}\right), j^{\prime}\right)=2$. By (6),

$$
\begin{aligned}
& \frac{\sum_{\tilde{i} \in \mathcal{S}_{1}(M(i))} P_{\tilde{i} j} P\left(s_{1}=\tilde{i} \mid s_{2}=j\right)}{\sum_{\tilde{i} \in \mathcal{S}_{1}(M(i))} P\left(s_{1}=\tilde{i} \mid s_{2}=j\right)} \leq \beta_{2} \leq \frac{\sum_{\tilde{i} \in \mathcal{S}_{1}\left(M\left(i^{\prime}\right)\right)} P_{\tilde{i} j} P\left(s_{1}=\tilde{i} \mid s_{2}=j\right)}{\sum_{\tilde{i} \in \mathcal{S}_{1}\left(M\left(i^{\prime}\right)\right)} P\left(s_{1}=\tilde{i} \mid s_{2}=j\right)}, \\
& \frac{\sum_{\tilde{i} \in \mathcal{S}_{1}(M(i))} P_{\tilde{i} j^{\prime}} P\left(s_{1}=\tilde{i} \mid s_{2}=j^{\prime}\right)}{\sum_{\tilde{i} \in \mathcal{S}_{1}(M(i))} P\left(s_{1}=\tilde{i} \mid s_{2}=j^{\prime}\right)} \geq \beta_{2} \geq \frac{\sum_{\tilde{i} \in \mathcal{S}_{1}\left(M\left(i^{\prime}\right)\right)} P_{\tilde{i} j^{\prime}} P\left(s_{1}=\tilde{i} \mid s_{2}=j^{\prime}\right)}{\sum_{\tilde{i} \in \mathcal{S}_{1}\left(M\left(i^{\prime}\right)\right)} P\left(s_{1}=\tilde{i} \mid s_{2}=j^{\prime}\right)} .
\end{aligned}
$$

Suppose $j<j^{\prime}$. Then, by Lemma 3 ,

$$
\begin{aligned}
\beta_{2} & \geq \frac{\sum_{\tilde{i} \in \mathcal{S}_{1}(M(i))} P_{\tilde{i} j} P\left(s_{1}=\tilde{i} \mid s_{2}=j\right)}{\sum_{\tilde{i} \in \mathcal{S}_{1}(M(i))} P\left(s_{1}=\tilde{i} \mid s_{2}=j\right)} \\
& >\frac{\sum_{\tilde{i} \in \mathcal{S}_{1}(M(i))} P_{\tilde{i} j^{\prime}} P\left(s_{1}=\tilde{i} \mid s_{2}=j^{\prime}\right)}{\sum_{\tilde{i} \in \mathcal{S}_{1}(M(i))} P\left(s_{1}=\tilde{i} \mid s_{2}=j^{\prime}\right)} \\
& \geq \beta_{2} .
\end{aligned}
$$


This is a contradiction. Similarly, suppose $j>j^{\prime}$. Then, Then, by Lemma 3,

$$
\begin{aligned}
\beta_{2} & \geq \frac{\sum_{\tilde{i} \in \mathcal{S}_{1}\left(M\left(i^{\prime}\right)\right)} P_{\tilde{i} j^{\prime}} P\left(s_{1}=\tilde{i} \mid s_{2}=j^{\prime}\right)}{\sum_{\tilde{i} \in \mathcal{S}_{1}\left(M\left(i^{\prime}\right)\right)} P\left(s_{1}=\tilde{i} \mid s_{2}=j^{\prime}\right)} \\
& >\frac{\sum_{\tilde{i} \in \mathcal{S}_{1}\left(M\left(i^{\prime}\right)\right)} P_{\tilde{i} j} P\left(s_{1}=\tilde{i} \mid s_{2}=j\right)}{\sum_{\tilde{i} \in \mathcal{S}_{1}\left(M\left(i^{\prime}\right)\right)} P\left(s_{1}=\tilde{i} \mid s_{2}=j\right)} \\
& \geq \beta_{2} .
\end{aligned}
$$

This is also a contradiction.

We now go back to the proof of Lemma 2. Lemma 4 implies $\mathcal{S}_{2}\left(i, i^{\prime}\right) \neq \emptyset \Rightarrow \mathcal{S}_{2}\left(i^{\prime}, i\right)=\emptyset$, which directly implies the former part of Lemma.

For the latter part, first, it is clear that $\mathcal{S}_{2}\left(i, i^{\prime}\right)=\mathcal{S}_{2}\left(i^{\prime}, i\right)=\emptyset$ implies $\mathbb{E}\left[u_{1}(t, A(M(i), j)) \mid s_{1}=i\right]=\mathbb{E}\left[u_{1}\left(t, A\left(M\left(i^{\prime}\right), j\right)\right) \mid s_{1}=i\right]$. Now suppose $\mathcal{S}_{2}\left(i, i^{\prime}\right) \neq \emptyset$ and $\mathcal{S}_{2}\left(i^{\prime}, i\right)=\emptyset$. Then we have

$$
\begin{aligned}
\mathbb{E} & {\left[u_{1}(t, A(M(i), j)) \mid s_{1}=i\right]-\mathbb{E}\left[u_{1}\left(t, A\left(M\left(i^{\prime}\right), j\right)\right) \mid s_{1}=i\right] } \\
& =\sum_{j \in \mathcal{S}_{2}\left(i, i^{\prime}\right)}\left[P\left(t=2, s_{2}=j \mid s_{1}=i\right)\left(1-b_{1}\right)-P\left(t=1, s_{2}=j \mid s_{1}=i\right)\left(1+b_{1}\right)\right] \\
& =2\left[\sum_{j \in \mathcal{S}_{2}\left(i, i^{\prime}\right)} P\left(s_{2}=j \mid s_{1}=i\right)\right]\left[\frac{\sum_{j \in \mathcal{S}_{2}\left(i, i^{\prime}\right)} P\left(t=2, s_{2}=j \mid s_{1}=i\right)}{\sum_{j \in \mathcal{S}_{2}\left(i, i^{\prime}\right)} P\left(s_{2}=j \mid s_{1}=i\right)}-\beta_{1}\right] \\
& =2\left[\sum_{j \in \mathcal{S}_{2}\left(i, i^{\prime}\right)} P\left(s_{2}=j \mid s_{1}=i\right)\right]\left[\frac{\sum_{j \in \mathcal{S}_{2}\left(i, i^{\prime}\right)} P\left(t=2, s_{1}=i, s_{2}=j\right)}{\sum_{j \in \mathcal{S}_{2}\left(i, i^{\prime}\right)} P\left(s_{1}=i, s_{2}=j\right)}-\beta_{1}\right] \\
& =2\left[\sum_{j \in \mathcal{S}_{2}\left(i, i^{\prime}\right)} P\left(s_{2}=j \mid s_{1}=i\right)\right]\left[\frac{\sum_{j \in \mathcal{S}_{2}\left(i, i^{\prime}\right)} \frac{P\left(t=2, s_{1}=i, s_{2}=j\right)}{P\left(s_{1}=i, s_{2}=j\right)} P\left(s_{1}=i, s_{2}=j\right)}{\sum_{j \in \mathcal{S}_{2}\left(i, i^{\prime}\right)} P\left(s_{1}=i, s_{2}=j\right)}-\beta_{1}\right] \\
& =2\left[\sum_{j \in \mathcal{S}_{2}\left(i, i^{\prime}\right)} P\left(s_{2}=j \mid s_{1}=i\right)\right]\left[\frac{\sum_{j \in \mathcal{S}_{2}\left(i, i^{\prime}\right)}\left(1-P P_{i j}\right) P\left(s_{1}=i, s_{2}=j\right)}{\sum_{j \in \mathcal{S}_{2}\left(i, i^{\prime}\right)} P\left(s_{1}=i, s_{2}=j\right)}-\beta_{1}\right] \\
& =2\left[\sum_{j \in \mathcal{S}_{2}\left(i, i^{\prime}\right)} P\left(s_{2}=j \mid s_{1}=i\right)\right]\left[\frac{\sum_{j \in \mathcal{S}_{2}\left(i, i^{\prime}\right)}\left(1-P_{i j}\right) P\left(s_{2}=j \mid s_{1}=i\right)}{\sum_{j \in \mathcal{S}_{2}\left(i, i^{\prime}\right)} P\left(s_{2}=j \mid s_{1}=i\right)}-\beta_{1}\right] .
\end{aligned}
$$


Similarly, suppose $\mathcal{S}_{2}\left(i, i^{\prime}\right)=\emptyset$ and $\mathcal{S}_{2}\left(i^{\prime}, i\right) \neq \emptyset$. Then we have

$$
\begin{aligned}
& \mathbb{E}\left[u_{1}(t, A(M(i), j)) \mid s_{1}=i\right]-\mathbb{E}\left[u_{1}\left(t, A\left(M\left(i^{\prime}\right), j\right)\right) \mid s_{1}=i\right] \\
& =\sum_{j \in \mathcal{S}_{2}\left(i^{\prime}, i\right)}\left[P\left(t=1, s_{2}=j \mid s_{1}=i\right)\left(1+b_{1}\right)-P\left(t=2, s_{2}=j \mid s_{1}=i\right)\left(1-b_{1}\right)\right] \\
& =2\left[\sum_{j \in \mathcal{S}_{2}\left(i^{\prime}, i\right)} P\left(s_{2}=j \mid s_{1}=i\right)\right]\left[\beta_{1}-\frac{\sum_{j \in \mathcal{S}_{2}\left(i^{\prime}, i\right)} P\left(t=2, s_{2}=j \mid s_{1}=i\right)}{\sum_{j \in \mathcal{S}_{2}\left(i^{\prime}, i\right)} P\left(s_{2}=j \mid s_{1}=i\right)}\right] \\
& =2\left[\sum_{j \in \mathcal{S}_{2}\left(i^{\prime}, i\right)} P\left(s_{2}=j \mid s_{1}=i\right)\right]\left[\beta_{1}-\frac{\sum_{j \in \mathcal{S}_{2}\left(i^{\prime}, i\right)} P\left(t=2, s_{1}=i, s_{2}=j\right)}{\sum_{j \in \mathcal{S}_{2}\left(i^{\prime}, i\right)} P\left(s_{1}=i, s_{2}=j\right)}\right] \\
& =2\left[\sum_{j \in \mathcal{S}_{2}\left(i^{\prime}, i\right)} P\left(s_{2}=j \mid s_{1}=i\right)\right]\left[\beta_{1}-\frac{\sum_{j \in \mathcal{S}_{2}\left(i^{\prime}, i\right)} \frac{P\left(t=2, s_{1}=i, s_{2}=j\right)}{P\left(s_{1}=i, s_{2}=j\right)} P\left(s_{1}=i, s_{2}=j\right)}{\sum_{j \in \mathcal{S}_{2}\left(i^{\prime}, i\right)} P\left(s_{1}=i, s_{2}=j\right)}\right] \\
& =2\left[\sum_{j \in \mathcal{S}_{2}\left(i^{\prime}, i\right)} P\left(s_{2}=j \mid s_{1}=i\right)\right]\left[\beta_{1}-\frac{\sum_{j \in \mathcal{S}_{2}\left(i^{\prime}, i\right)}\left(1-P_{i j}\right) P\left(s_{1}=i, s_{2}=j\right)}{\sum_{j \in \mathcal{S}_{2}\left(i^{\prime}, i\right)} P\left(s_{1}=i, s_{2}=j\right)}\right] \\
& =2\left[\sum_{j \in \mathcal{S}_{2}\left(i^{\prime}, i\right)} P\left(s_{2}=j \mid s_{1}=i\right)\right]\left[\beta_{1}-\frac{\sum_{j \in \mathcal{S}_{2}\left(i^{\prime}, i\right)}\left(1-P_{i j}\right) P\left(s_{2}=j \mid s_{1}=i\right)}{\sum_{j \in \mathcal{S}_{2}\left(i^{\prime}, i\right)} P\left(s_{2}=j \mid s_{1}=i\right)}\right] \text {. }
\end{aligned}
$$

Proof of Theorem 1: Suppose to the contrary that there exists an informative equilibrium. Then $M(i)=i$ for any $i \in S_{1}$ and there exists some $j_{1}$ on which the sender's message is influential. We then define $j^{*}, \bar{i}^{*}$, and $\underline{i}^{*}$ by the following procedure:

Step 0: Go to Step 1-1 with $j_{1}$.

Step $k$-1: Set $\bar{i}_{k}=i\left(j_{k}\right)$ and go to Step $k-2$.

Step $k$-2: Set $\underline{i}_{k}=\max \left\{i \mid A\left(i, j_{k}\right)=1\right\}$ and go to Step $k$-3.

Step $k$-3 If $j_{k}=\max \mathcal{S}_{2}\left(\bar{i}_{k}, \underline{i}_{k}\right)$, the set $j^{*}=j_{k}, \bar{i}^{*}=\bar{i}_{k}, \underline{i}^{*}=\underline{i}_{k}$ and stop the process. If $j_{k} \neq \max \mathcal{S}_{2}\left(\bar{i}_{k}, \underline{i}_{k}\right)$, then set $j_{k+1}=\max \mathcal{S}_{2}\left(\bar{i}_{k}, \underline{i}_{k}\right)$ and go to Step $(k+1)-1$.

For any $k, j_{k} \leq j_{k+1}$ (if any) since $j_{k} \in \mathcal{S}_{2}\left(\bar{i}_{k}, \underline{i}_{k}\right)$. Then the above procedure and $j^{*}, \bar{i}^{*}$, and $\underline{i}^{*}$ are well-defined. Of course, it is satisfied $\vec{i}^{*}=i\left(j^{*}\right)$ and $j^{*}=\max \mathcal{S}_{2}\left(\vec{i}^{*}, \underline{i}^{*}\right)$. The former fact implies that $P_{\bar{i}^{*} j^{*}}>1-\beta_{1}$ and $A\left(\bar{i}^{*}, j^{*}\right)=2$. By the latter fact and (5), it follows that 
for any $j \in \mathcal{S}_{2}\left(\bar{i}^{*}, \underline{i}^{*}\right)$,

$$
P_{\bar{i}^{*} j} \geq P_{\bar{i}^{*} j^{*}}>1-\beta_{1}
$$

This violates (ii) of (7). This is a contradiction.

Proof of Theorem 2: Consider $M$ that is truth telling and $A$ that satisfies (6). Since there is no tie-breaking under Condition 2, such $A$ is uniquely determined. Then, Lemma 1 and (ii) of Condition 2 imply that $(M, A)$ is influential as long as it is an equilibrium.

(6) implies that for any $j \in \mathcal{S}_{2}\left(i^{\prime}, i\right), P_{i j} \geq \beta_{2}$, which in turn implies

$$
1-P_{i j} \leq 1-\beta_{2}<\frac{1}{2}<\beta_{1}
$$

It follows that (iii) of (7) always holds. Then, it suffices to show that (ii) of (7) holds. Fix $i$ and $i^{\prime}$ such that $\mathcal{S}_{2}\left(i, i^{\prime}\right) \neq \emptyset$. By (6) and (i) of Condition 2 , for any $j \in \mathcal{S}_{2}\left(i, i^{\prime}\right), P_{i j}<1-\beta_{1}$ holds. It follows that (ii) of (7) holds. This completes the proof.

Proof of Proposition 3: (5) and Proposition 1 directly imply the following results (the proofs are omitted).

Lemma 5 In equilibrium, for any $j$, there exists $\bar{i}(j) \in\{0\} \cup S_{1}$ such that

$$
A(M(i), j)= \begin{cases}1 & \text { if } i \leq \bar{i}(j) \\ 2 & \text { if } i \geq \bar{i}(j)+1\end{cases}
$$

Moreover, $j<j^{\prime}$ implies $\bar{i}(j) \geq \bar{i}\left(j^{\prime}\right)$.

Lemma 6 Suppose that there exists an equilibrium $(M, A)$ in which there exist $i, i^{\prime}, m$, and $m^{\prime}$ such that

$$
\begin{aligned}
& i<i^{\prime}, \\
& M(i)=m \neq m^{\prime}=M\left(i^{\prime}\right), \\
& A(m, j)=A\left(m^{\prime}, j\right) \quad \forall j .
\end{aligned}
$$


Then, there is also an equilibrium $(\hat{M}, A)$ such that

$$
\hat{M}(\tilde{i})= \begin{cases}m & \text { if } \tilde{i}=i^{\prime}, \\ M(\tilde{i}) & \text { otherwise. }\end{cases}
$$

The proposition follows from these results.

Proof of Theorem 3: Suppose to the contrary that there exists an equilibrium in which the sender's message is influential. By Proposition 3, there must be an equilibrium in which there exist $i_{1}, i_{2} \in S_{1}, j_{1}, j_{2} \in S_{2}$, and $m_{1}, m_{2} \in M$ such that

$$
\begin{aligned}
& 1 \leq i_{1}<i_{2} \leq\left|S_{1}\right| \\
& 1 \leq j_{1}<j_{2} \leq\left|S_{2}\right|, \\
& m_{1} \neq m_{2}, \\
& M\left(s_{1}\right)= \begin{cases}m_{1} & \text { iff } 1 \leq s_{1} \leq i_{1}, \\
m_{2} & \text { iff } i_{1}+1 \leq s_{1} \leq i_{2},\end{cases} \\
& A\left(m, s_{2}\right)=\left\{\begin{array}{lll}
1 \quad \text { if } \quad & m=m_{1} \text { and } 1 \leq s_{2} \leq j_{2}, \text { or } \\
& m=m_{2} \text { and } 1 \leq s_{2} \leq j_{1}, \\
2 \text { if } \quad & m=m_{1} \text { and } j_{2}+1 \leq s_{2} \leq\left|S_{2}\right|, \text { or } \\
& m=m_{2} \text { and } j_{1}+1 \leq s_{2} \leq\left|S_{2}\right| .
\end{array}\right.
\end{aligned}
$$

Considering Player 2's incentive on receiving $s_{2}=j_{2}$, (5) and Lemma 1 require that $j_{2} \leq \hat{j}$ should hold. On the other hand, considering Player 1's incentive on receiving $s_{1}=i_{1}+1$, (5) and Lemma 2 require that $j_{2}>\hat{j}$ should hold. This is a contradiction. 


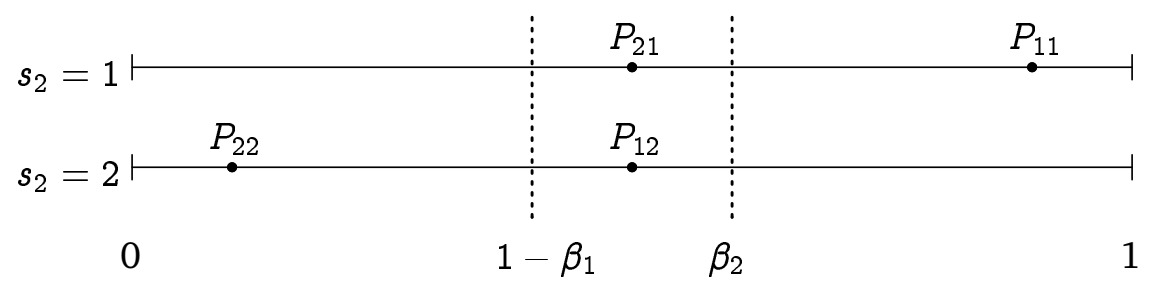

Figure 1: The signal structures that satisfy Condition 1.

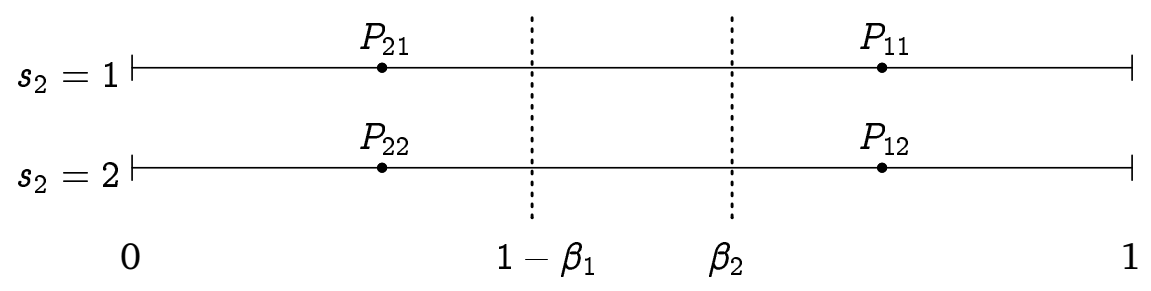

Figure 2: The signal structures that satisfy Condition 2. 